\title{
Use of Typhoid Vi-Polysaccharide Vaccine as a Vaccine Probe to Delineate Clinical Criteria for Typhoid Fever
}

\author{
Md. Taufiqul Islam, ${ }^{1} \dagger$ Justin Im, ${ }^{2 *}$ † Faisal Ahmmed, ${ }^{1}$ Deok Ryun Kim, ${ }^{2}$ Ashraful Islam Khan, ${ }^{1}$ Khalequ Zaman, ${ }^{1}$ Mohammad Ali, ${ }^{3}$ \\ Florian Marks, ${ }^{2,4}$ Firdausi Qadri, ${ }^{1}$ Jerome H. Kim, ${ }^{2}$ and John D. Clemens ${ }^{1,5,6}$ \\ ${ }^{1}$ International Centre for Diarrheal Disease Research, Bangladesh, Dhaka, Bangladesh; ${ }^{2}$ International Vaccine Institute, Seoul, Republic of \\ Korea; ${ }^{3}$ Johns Hopkins University, Baltimore, Maryland; ${ }^{4}$ Department of Medicine, University of Cambridge, Cambridge, United Kingdom; \\ ${ }^{5}$ UCLA Fielding School of Public Health, Los Angeles, California; ${ }^{6}$ Korea University College of Medicine, Seoul, South Korea
}

\begin{abstract}
Blood cultures (BCs) detect an estimated $50 \%$ of typhoid fever cases. There is need for validated clinical criteria to define cases that are BC negative, both to help direct empiric antibiotic treatment and to better evaluate the magnitude of protection conferred by typhoid vaccines. To derive and validate a clinical rule for defining BC-negative typhoid fever, we assessed, in a cluster-randomized effectiveness trial of Vi-polysaccharide (ViPS) typhoid vaccine in Kolkata, India, 14,797 episodes of fever lasting at least 3 days during 4 years of comprehensive, BC-based surveillance of 70,865 persons. A recursive partitioning algorithm was used to develop a decision rule to predict BC-proven typhoid cases with a diagnostic specificity of $97-98 \%$. To validate this rule as a definition for BC-negative typhoid fever, we assessed whether the rule defined culture-negative syndromes prevented by ViPS vaccine. In a training subset of individuals, we identified the following two rules: rule 1: patients aged $<15$ years with prolonged fever accompanied by a measured body temperature $\geq 100^{\circ} \mathrm{F}$, headache, and nausea; rule 2: patients aged $\geq 15$ years with prolonged fever accompanied by nausea and palpable liver but without constipation. The adjusted protective efficacy of ViPS against clinical typhoid defined by these rules in persons aged $\geq 2$ years in a separate validation subset was 33\% (95\% Cl: $4-53 \%)$. We have defined and validated a clinical rule for predicting BC-negative typhoid fever using a novel vaccine probe approach. If validated in other settings, this rule may be useful to guide clinical care and to enhance typhoid vaccine evaluations.
\end{abstract}

\section{INTRODUCTION}

Typhoid fever is a poverty-related disease, with a high disease burden estimated at 14.3 million cases and approximately 200,000 deaths per year globally ${ }^{1}$ found disproportionately in low- and middle-income countries. ${ }^{2,3}$ Unfortunately, the standard diagnostic test, culture of blood, is only about $50 \%$ sensitive in detecting typhoid fever, ${ }^{4-7}$ and there are neither reliable point-of-care diagnostic tests nor well-validated rules for defining blood culture (BC)-negative typhoid using combinations of presenting clinical signs and symptoms to guide empiric antibiotic therapy nor to permit complete evaluation of the preventive impact of typhoid vaccines. ${ }^{8,9}$ The need for more selective targeting of patients for antibiotic therapy is underscored by growing antibiotic resistance and the need for antibiotic stewardship. ${ }^{10-12}$ Similarly, the need for more comprehensive assessments of prevented typhoid cases is highlighted by the advent of improved typhoid vaccines now under clinical evaluation. ${ }^{13-17}$

One of the challenges confronting development of non-BCbased criteria for typhoid fever is the absence of gold standard diagnostic tests for such cases. For several other invasive bacterial diseases, including invasive Haemophilus influenzae type $b$ and Streptococcus pneumoniae, vaccine probe studies have been used to define pathogen-attributable disease syndromes in which cultures of normally sterile fluids are negative. ${ }^{18-22}$ The probe design, which relies on detecting vaccine efficacy against these culture-negative clinical syndromes, can produce an estimate of the aetiological fraction of a disease syndrome because of the pathogen targeted by vaccine, effectively overcoming challenges in diagnostic

\footnotetext{
*Address correspondence to Justin Im, International Vaccine Institute, SNU Research Park, 1-Gwanak-ro, Gwanak-gu, Seoul, 08826, Republic of Korea. E-mail: justin.im@ivi.int

†These authors contributed equally to this work.
}

insensitivity. ${ }^{22}$ However, this approach only works when a clinical syndrome can be defined for which the proportion of culture-negative disease caused by the target pathogen is sufficiently high. If low, measured vaccine protection will be "washed out" by cases not caused by the target pathogen.

In an earlier cluster-randomized trial (CRT) of typhoid Vipolysaccharide (ViPS) vaccine, it was possible to detect both direct and herd protection of Vi against BC-proven typhoid. ${ }^{23}$ However, it was not possible to detect significant Vi protection, either direct or herd, against BC-negative cases presenting with fever of at least 3 days duration, suggesting that we needed more specific clinical criteria for defining the target syndrome than prolonged fever per se. However, we lacked these clinical criteria. In this article, we present a novel approach for defining clinical criteria for culture-negative, clinical typhoid leveraging a vaccine probe analysis within the earlier CRT of the ViPS vaccine.

\section{METHODS AND MATERIALS}

General approach. We reanalyzed a CRT of ViPS vaccine launched in 2004, as well as 2 years of antecedent typhoid surveillance in the vaccine trial population, in urban slums in Kolkata, India. ${ }^{23}$ To derive an empirically validated clinical rule for BC-negative, clinical typhoid fever, we required that three conditions be met: 1) the clinical features for the rule should also discriminate between episodes of prolonged fever that are BC positive for $S$. Typhi and those that are negative for $S$. Typhi, 2) the diagnostic specificity of the rule should yield a number of BC-negative clinical typhoid episodes approximately equal to the number of BC-positive episodes in the same population (because BCs are known to be about $50 \%$ sensitive in detected typhoid fever), ${ }^{24-28}$ and 3 ) a rule meeting the first two conditions should identify BC-negative episodes against which there was detectable vaccine efficacy by ViPS vaccine in the CRT, thereby using ViPS vaccine as a probe. To 
derive a clinical rule with the desired level of diagnostic specificity, we used recursive partitioning, a classification algorithm that considers candidate individual variables for the rule conjointly to arrive at a rule with desired levels of specificity and sensitivity. ${ }^{29}$ This approach has several advantages over other machine learning classification algorithms, namely, that it produces an easily visualized decision tree allowing for simplistic interpretation of subject classification.

The ViPS vaccine trial in Kolkata. The study was conducted in an urban slum site in Kolkata city, the capital of West Bengal, India. The Kolkata Municipal Corporation area had an estimated population of six million at the time of the trial. A census of 66,458 people was conducted 2 years before the onset of the trial. Individual- and household-level demographic and socioeconomic data were subsequently updated at regular intervals in a demographic surveillance system that continued through the vaccine trial. A close-out census was carried out at the end of surveillance, 2 years after vaccination. Typhoid fever surveillance was carried out in the study population during a 2-year lead-in period before vaccination and continued for 2 years following vaccination. Subjects from the study area who presented at one of five study clinics with a history of fever for at least 3 days were enrolled by a study physician. Subjects' clinical histories and physical findings were systematically captured during enrollment. After obtaining verbal informed consent, a 5-mL blood specimen was collected by venipuncture for microbiological culture using an automated system (Becton Dickinson, Franklin Lakes, NJ) and bacterial identification through conventional biochemical and serologic methods. ${ }^{30}$ The CRT was initiated in 2004. Residents aged 2 years and older in 80 clusters were randomly allocated at a one-to-one ratio to Vi vaccine (Typherix, GlaxoSmithKline, Brentford, United Kingdom) or inactivated hepatitis A (HepA) vaccine (Havrix, GlaxoSmithKline). A total of 18,869 participants received $\mathrm{Vi}$ vaccine, and 18,804 received HepA vaccine. After 2 years of surveillance, the total Vi-vaccine protection against typhoid fever (comparing the incidence of typhoid in Vi vaccinees versus HepA vaccinees) was $61 \%$; indirect protection (comparing the incidence of typhoid in non-vaccinees in the Vi clusters versus non-vaccinees in the HepA clusters) and overall protection (comparing the incidence of typhoid in all members of the Vi clusters versus all members of the HepA clusters) were $44 \%$ and $57 \%$, respectively. ${ }^{23}$

Definition and assembly of prolonged fever episodes for analysis. We assembled all prolonged febrile episodes from the surveillance leading up to and during the clusterrandomized trial. A febrile visit was defined as an individual visit by a study area resident to a study clinic presenting with a history of fever for at least 3 days. A febrile episode was defined as all component febrile visits in which the onset of symptoms for a subsequent visit was less than 14 days from the date of discharge for the previous visit. A BC-proven typhoid episode was considered to be a febrile episode in which Salmonella enterica serovar Typhi (S. Typhi) was isolated from at least one BC. For this analysis, where multiple fever visits comprised a febrile episode, we considered the date of onset of illness to be the date of onset of symptoms for the first component visit of the episode. From these episodes, analysis included individuals for whom data for all clinical parameters under consideration (vide infra) were available (Figure 1).

Statistical analysis. We used a machine learning algorithm to create a decision tree constructed via binary recursive partitioning. ${ }^{31}$ The decision tree was designed to predict whether a febrile episode was culture-positive typhoid fever, using 22 clinical signs and symptoms and epidemiological characteristics recorded at the time of initial presentation for care for the episode. Each sign and symptom was first converted into a dichotomous variable. For the machine learning modeling, we selected as candidate variables only those that had an association with typhoid with $P$ value $\leq 0.25$ in bivariate logistic regression analyses.

Because predictive rules may overestimate predictive performance when tested on the data used to derive the rules, we split all individuals who resided in the study area during the 4 years of follow-up, at random, into two approximately equal-sized groups, for development (training dataset) and validation (validation dataset) of the clinical predictive rule, respectively. For development and testing of the rule, we considered all febrile episodes of all patients presenting with

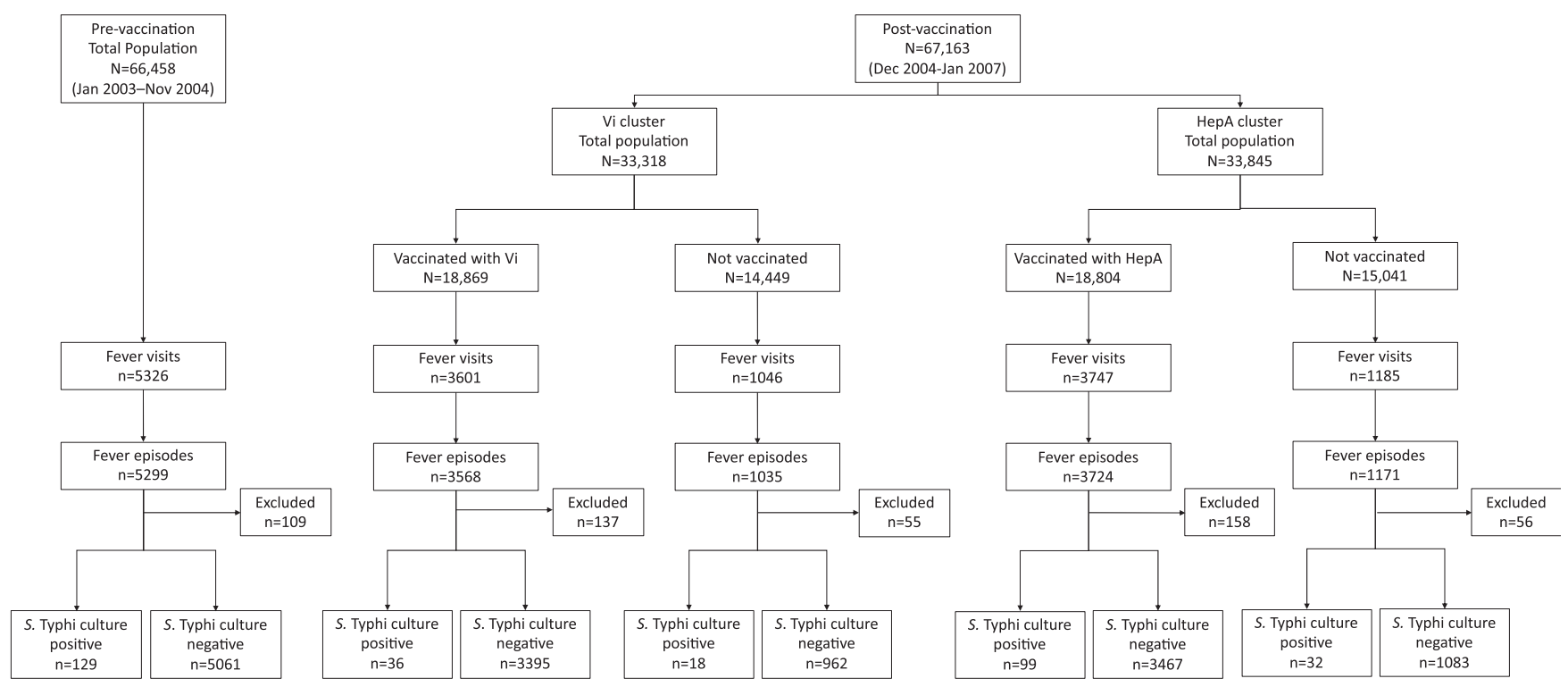

FigURE 1. Flow diagram showing culture-confirmed typhoid fever in Vi and HepA clusters. 
at least 3 days of fever in the training and validation groups for whom complete clinical data were available. We initially ran the recursive partitioning algorithm for candidate clinical predictive variables in the training set episodes, specifying an alternative loss function that penalized a false-negative prediction 10 times more heavily than a false-positive prediction. Because of unbalanced class data, we set the minimum number of observations as 30 for the nonterminal nodes of the decision tree and 10 for the terminal nodes of the decision tree. For cross-validation, the training set was randomly partitioned into 10 parts, one part was used in estimating cross-validation error. ${ }^{32}$

For these analyses of the training dataset, we constructed trees at several different levels, choosing the tree with the lowest level, and thus the simplest clinical algorithm, that still met our three performance criteria (vide supra). To find the optimal tree in the training set, the model was pruned by the minimal complexity parameter corresponding to minimum error with at least five tree rules. We constructed a receiver operating characteristic (ROC) curve for the selected rule, evaluating the area under the ROC curve as described elsewhere, ${ }^{33,34}$ and also used the curve to identify a cutoff probability that corresponded to the highest sensitivity for cutoffs, giving $97-98 \%$ specificity. To measure the protective efficacy (PE) of ViPS vaccine against culture-negative typhoid, we contrasted ViPS vaccine recipients with HepA vaccine recipients for the occurrence of all episodes of culturenegative fever meeting the criteria of the clinical prediction rule, using the Andersen-Gill (AG) generalized Cox proportional hazard regression model after verifying that the proportionality assumptions were fulfilled. ${ }^{35}$ The model was adjusted for age of the individual and living in a household that used a designated place for waste disposal. The hazard ratio (HR) for culture-negative typhoid fever was estimated by exponentiation of the coefficient of receipt of ViPS in the model, and the $95 \% \mathrm{Cl}$ for the HR was estimated using a robust sandwich method. The protective effectiveness (PE) of ViPS vaccination against BC-negative typhoid fever defined by our clinical rule was estimated as $(1-\mathrm{HR}) \times 100 \%$. We considered $P<0.05$ (two-tailed) as the margin of statistical significance. The analysis was performed using "rpart" package for decision tree modeling, "caTools" for random selection, "rpart.plot" package for tree plotting, "pROC" packages for ROC curve, ${ }^{36}$ "survival" for AG Cox model, ${ }^{37}$ and "dplyr" package for data management under R-Studio. ${ }^{38}$ Once having conducted these developmental analyses in the training dataset, we assessed the sensitivity and specificity of the rule in predicting BC-positive typhoid fever and in defining a BC-negative syndrome against which ViPS protected in the validation dataset.

\section{RESULTS}

From March 2003 to January 2007, a total population of 70,865 individuals was included in the surveillance. During vaccination, which took place between November 2004 and December 2004, 67,163 age-eligible individuals were randomized to receive either ViPS vaccine (18,869 vaccinated) or HepA vaccine $(18,804$ vaccinated) (Figure 1). Total protection by ViPS was $61 \%$ (95\% Cl: $41-75 ; P<0.001)$; complete results of the vaccine trial have been reported previously. ${ }^{23} \mathrm{~A}$ total of 14,905 febrile visits, comprising 14,797 fever episodes, in 11,255 individuals were observed between March 19, 2003 and January 28, 2007, from which 323 BC-proven typhoid cases were recorded. For our analysis, 515 fever episodes, including nine typhoid cases, were excluded due to incompatibility with fever criterion (i.e., fever duration less than 3 days) or incomplete clinical metadata (Figure 1). We included 14,282 fever episodes and 314 $\mathrm{BC}$-proven typhoid cases in the analysis. From bivariate logistic regression analysis, 17 of 22 recorded clinical and epidemiological features had a significant association $(P$-value $\leq 0.25)$ with confirmed typhoid. These include age $<15$ years, typhoid season (April to June and September to November), high temperature ( $\geq 100^{\circ} \mathrm{F}$ by axillary measurement), abdominal pain, constipation,

TABLE 1

Bivariate relationships ${ }^{*}$ between candidate clinical and epidemiological features and typhoid fever

\begin{tabular}{|c|c|c|c|}
\hline Feature & Culture-negative fever episode $(n=13,968), n(\%)$ & Culture-positive typhoid fever episode $(n=314), n(\%)$ & $P$-value \\
\hline Age $(<15$ years $) \dagger$ & 4,742 (33.9) & $191(60.8)$ & $<0.001$ \\
\hline Gender (male) & $7,180(52.1)$ & $159(51.8)$ & 0.919 \\
\hline Typhoid season $\ddagger$ & $7,573(54.2)$ & $201(64.0)$ & 0.001 \\
\hline Fever duration ( $>7$ days)§ & $1,238(8.9)$ & $28(8.9)$ & 0.973 \\
\hline High temperature $\left(\geq 100^{\circ} \mathrm{F}\right) \|$ & 7,012 (50.2) & 205 (65.3) & $<0.001$ \\
\hline Abdominal pain & $1,711(12.2)$ & $71(22.6)$ & $<0.001$ \\
\hline Constipation & 1,752 (12.5) & 47 (15.0) & 0.201 \\
\hline Dehydration & $13,134(94)$ & $284(90.4)$ & 0.009 \\
\hline Diarrhea & $374(2.7)$ & $12(3.8)$ & 0.219 \\
\hline Abdominal distension & 709 (5.1) & $24(7.6)$ & 0.043 \\
\hline Drowsiness & $110(0.8)$ & $8(2.5)$ & 0.001 \\
\hline Headache & $8,015(57.4)$ & $195(62.1)$ & 0.095 \\
\hline Ileus sign & $5(0)$ & $0(0)$ & 0.967 \\
\hline Nausea & 3,312 (23.7) & $95(30.3)$ & 0.007 \\
\hline Palpable liver & $577(4.1)$ & $22(7)$ & 0.013 \\
\hline Peritoneal signs & $6(0)$ & $0(0)$ & 0.964 \\
\hline Seizure & $19(0.1)$ & $0(0)$ & 0.972 \\
\hline Palpable spleen & 267 (1.9) & $9(2.9)$ & 0.228 \\
\hline Abdominal tenderness & $1,079(7.7)$ & $36(11.5)$ & 0.015 \\
\hline Heart rate (> 80 bpm) & $12,054(86.3)$ & 299 (95.2) & $<0.001$ \\
\hline Thirsty & $3,455(24.7)$ & $117(37.3)$ & $<0.001$ \\
\hline Vomiting & $1,341(9.6)$ & $42(13.4)$ & 0.026 \\
\hline
\end{tabular}

$\dagger$ Age was considered at the date of fever onset.

$\ddagger$ Typhoid seasons defined as April to June and September to November.

$\S$ By reported clinical history.

\| Axillary, oral, or rectal temperature measurement. 
TABLE 2

Population assembly for training and validation sets

\begin{tabular}{|c|c|c|c|c|c|c|c|c|c|}
\hline & & & \multirow{2}{*}{$\frac{\text { Population }}{N}$} & \multicolumn{2}{|c|}{ Full study period } & \multicolumn{2}{|c|}{ Before vaccination } & \multicolumn{2}{|c|}{ After vaccination } \\
\hline & & & & Fever episodes (subjects) & Typhoid cases & Fever episodes & Typhoid cases & Fever episodes & Typhoid \\
\hline \multirow[t]{6}{*}{ Full set } & \multicolumn{2}{|c|}{ Before vaccination } & 3,702 & 102 & 4 & 102 & 4 & & \\
\hline & \multirow[t]{2}{*}{ Vi cluster } & Vaccinated & 18,869 & 5,438 & 91 & 2,007 & 55 & 3,431 & 36 \\
\hline & & Not vaccinated & 14,449 & 1,459 & 26 & 479 & 8 & 980 & 18 \\
\hline & \multirow[t]{3}{*}{ HepA cluster } & HepA vaccinated & 18,804 & 5,639 & 147 & 2,073 & 48 & 3,566 & 99 \\
\hline & & Not vaccinated & 15,041 & 1,644 & 46 & 529 & 14 & 1,115 & 32 \\
\hline & & Total & 70,865 & $14,282(10,924)$ & 314 & 5,190 & 129 & 9,092 & 185 \\
\hline \multirow{6}{*}{$\begin{array}{l}\text { Training } \\
\text { set }\end{array}$} & \multicolumn{2}{|c|}{ Before vaccination } & 1,851 & 56 & 2 & 56 & 2 & & \\
\hline & \multirow[t]{2}{*}{ Vi cluster } & Vaccinated & 9,436 & 2,752 & 41 & 1,004 & 21 & 1,748 & 20 \\
\hline & & Not vaccinated & 7,197 & 732 & 11 & 217 & 4 & 515 & 7 \\
\hline & \multirow[t]{3}{*}{ HepA cluster } & HepA vaccinated & 9,404 & 2,846 & 77 & 1,047 & 31 & 1,799 & 46 \\
\hline & & Not vaccinated & 7,540 & 809 & 26 & 257 & 11 & 552 & 15 \\
\hline & & Total & 35,428 & $7,195(5,462)$ & 157 & 2,581 & 69 & 4,614 & 88 \\
\hline \multirow{6}{*}{$\begin{array}{l}\text { Validation } \\
\text { set }\end{array}$} & \multicolumn{2}{|c|}{ Before vaccination } & 1,851 & 46 & 2 & 46 & 2 & & \\
\hline & \multirow[t]{2}{*}{ Vi cluster } & Vi vaccinated & 9,433 & 2,686 & 50 & 1,003 & 34 & 1,683 & 16 \\
\hline & & Not vaccinated & 7,252 & 707 & 13 & 262 & 4 & 465 & 11 \\
\hline & \multirow[t]{3}{*}{ HepA cluster } & HepA vaccinated & 9,400 & 2,793 & 70 & 1,026 & 17 & 1,767 & 53 \\
\hline & & Not vaccinated & 7,501 & 816 & 20 & 272 & 3 & 563 & 17 \\
\hline & & Total & 35,437 & $7,087(5,462)$ & 157 & 2,609 & 60 & 4,478 & 97 \\
\hline
\end{tabular}

Note: For randomized individuals who did not receive a vaccine, vaccine date was set as the median date of vaccination of vaccinees in the cluster of residence.

dehydration, diarrhea, abdominal distension, drowsiness, headache, nausea, palpable liver, palpable spleen, abdominal tenderness, heart rate $>80 /$ minute, thirst, and vomiting (Table 1). Blood culture-confirmed typhoid was not observed among individuals exhibiting abdominal peritoneal signs or seizure.

Assembly of training and validation datasets. In the training dataset, 7,195 febrile episodes in 5,462 individuals were randomly selected. Among these episodes, 1,748 and 1,799 episodes were from ViPS vaccine and HepA vaccine recipients, respectively, occurred after vaccination. The rest of the episodes were from the nonparticipants in the trial. Similarly, 7,087 febrile episodes from the 5,462 individuals were randomly selected for the validation set, of which 1,683 and 1,767 episodes were from ViPS vaccine and HepA vaccine recipients, respectively, occurred after vaccination (Table 2).
Analysis of training dataset. The training set was used to build a training model to predict BC-proven typhoid. The model was tuned by varying cutoff probabilities starting from $0 \%$ to $50 \%$ incremented by $1 \%$ using the grid-search technique, ${ }^{39}$ and the predefined specificity (97-98\%) was matched at $6-10 \%$ cutoff probabilities. A decision tree with a depth of four was selected as the optimal model because it was able to provide a sufficiently simple rule for clinical diagnosis and yielded specified probability cutoff range of $9 \%$. We derived the following rules for defining BC-proven typhoid in the population (Figure 2).

Rule 1: patients aged $<15$ years with high temperature $\left(\geq 100^{\circ} \mathrm{F}\right)$, headache, and nausea.

Rule 2: patients aged $\geq 15$ years with nausea, palpable liver, and without constipation.

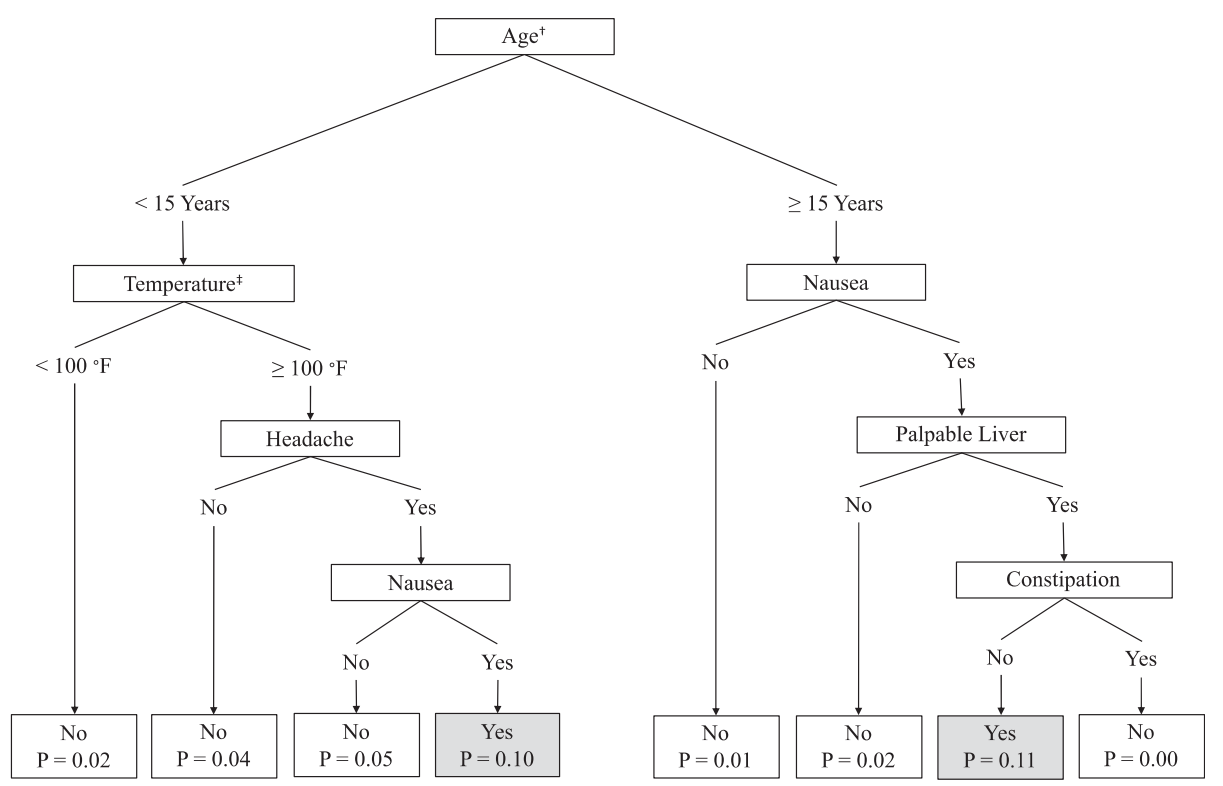

FIGURE 2. Depth four decision tree specifying clinical and epidemiological criteria for prediction of blood culture-positive typhoid. Note: Prediction (represented by terminal node) cutoff probability $(P)$ is set as 0.09 . $\dagger$ Age was considered at date of fever onset. $\ddagger$ Axillary, oral, or rectal temperature measurement. 


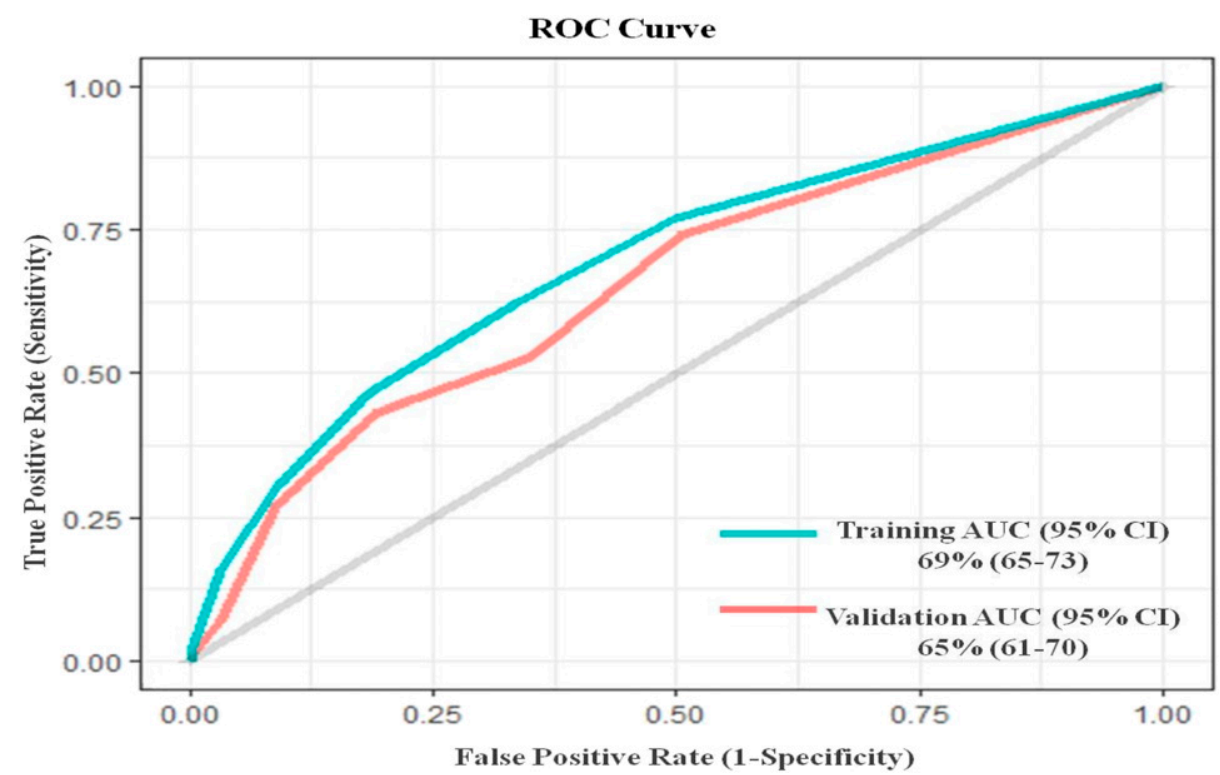

FIGURE 3. Receiver operating characteristic curve for different cutoff values for defining typhoid in the training and validation datasets. AUC $=$ area under the curve. This figure appears in color at www.ajtmh.org.

Overall, the accuracy of the training model was 95\%, with $16 \%$ sensitivity and $97 \%$ specificity, and the area under curve (AUC) showed significant difference from the $50 \%$ random line, measured as $69 \%$ (95\% Cl: 65-73) (Figure 3). When these criteria were used to define $\mathrm{BC}$-negative syndromes, the adjusted PE of the ViPS vaccine was $31 \%(95 \% \mathrm{Cl}: 2-52, P=0.040)$ for all age-groups (Table 3). Evaluations of the validation dataset yielded similar results. Overall, the accuracy of the validation model was $95 \%$, with $97 \%$ specificity. The sensitivity of the model decreased from $16.0 \%$ to $7.0 \%$; however, the AUC from the ROC curve remained significant at 65\% (95\% Cl: 61-70) (Figure 3). The vaccine PE of ViPS vaccine against BC-negative syndromes meeting the derived clinical criteria in the validation set was 33\% (95\% Cl: 4-53, $P=0.027)$ for all age-groups. By comparison, the PE of ViPS vaccine against all fever episodes was $0 \%$ (95\% Cl: -7 to 7$)$ and $1 \%(95 \% \mathrm{Cl}:-6$ to 8$)$ in the training and validation datasets, respectively (Supplemental Table 1).

\section{DISCUSSION}

Our analysis demonstrates that it is possible to use clinical, demographic, and epidemiological variables to describe a clinical syndrome for typhoid in a manner which maintains acceptable diagnostic specificity for BC-proven typhoid, yielding a plausible number of clinical typhoid cases in the $\mathrm{BC}$ negative group, and demarcates a group of BC-negative, persistent febrile episodes against which ViPS vaccine protects. We believe that this is the first time that an effective typhoid vaccine has been used as a "probe" to test the validity of clinical criteria for clinical typhoid.

Several studies have investigated the clinical symptoms predictive of proven typhoid fever, although these studies assessed disparate populations and age-groups, include varying ranges of clinical factors, and used different methods to substantiate their findings. Despite this heterogeneity, several notable variables are stated to have a high positive predictive value for culture-confirmed typhoid or to be statistically associated with culture-confirmed typhoid by logistic regression across multiple studies. These clinical variables include fever, ${ }^{40-44}$ relative bradycardia, ${ }^{45-47}$ coated tongue, ${ }^{46,47}$ positive Widal test, ${ }^{44,47}$ vomiting, ${ }^{40-43}$ diarrhea, ${ }^{40-43}$ palpable spleen, ${ }^{40-44}$ and palpable liver. ${ }^{42-44,48}$ A clinical trial of ViPS vaccine in Nepal identified culture-negative patients with clinical suspicion of typhoid fever (defined as prolonged fever of three

TABLE 3

Protective effectiveness of Vi-polysaccharide vaccine against clinical typhoid as defined by rules 1 and 2

\begin{tabular}{|c|c|c|c|c|c|c|c|c|c|c|c|c|}
\hline & \multicolumn{4}{|c|}{ Vi vaccine group } & \multicolumn{4}{|c|}{ HepA vaccine group } & \multicolumn{3}{|c|}{ Protective effectiveness (PE) } & \multirow[b]{2}{*}{$P$-value } \\
\hline & N & 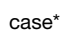 & PY & IR per $10^{5} \mathrm{PY}$ & N & case $^{\star}$ & PY & IR per $10^{5} \mathrm{PY}$ & Crude PE & $P$-value & Adj. PE & \\
\hline \multicolumn{13}{|l|}{ Training set } \\
\hline All ages (years) & 9,436 & 51 & 18,345 & 278 & 9,404 & 77 & 18,266 & 422 & $34(6,54)$ & 0.021 & $31(2,52) \dagger$ & 0.040 \\
\hline $2-14$ & 2,657 & 49 & 5,143 & 953 & 2,847 & 74 & 5,511 & 1,343 & $29(-2,51)$ & 0.062 & $29(-2,50) \ddagger$ & 0.063 \\
\hline$\geq 15$ & 6,779 & 2 & 13,202 & 15 & 6,557 & 3 & 12,754 & 24 & $36(-285,89)$ & 0.630 & $36(-285,89) \ddagger$ & 0.630 \\
\hline \multicolumn{13}{|l|}{ Validation set } \\
\hline All ages (years) & 9,433 & 52 & 18,320 & 284 & 9,400 & 79 & 18,242 & 433 & $34(7,54)$ & 0.018 & $33(4,53) \dagger$ & 0.027 \\
\hline $2-14$ & 2,722 & 49 & 5,284 & 927 & 2,832 & 75 & 5,486 & 1,367 & $32(3,53)$ & 0.035 & $32(3,53) \ddagger$ & 0.033 \\
\hline$\geq 15$ & 6,711 & 3 & 13,035 & 23 & 6,568 & 4 & 12,756 & 31 & $27(-227,84)$ & 0.684 & $27(-227,84) \ddagger$ & 0.684 \\
\hline
\end{tabular}

*Based on clinical typhoid end point, defined as either rule 1 (patients aged $<15$ years with high temperature $\left(\geq 100^{\circ} \mathrm{F}\right)$, headache, and nausea) or rule 2 (patients aged $\geq 15$ years with nausea, palpable liver, and without constipation).

†Protective effectiveness is adjusted for age and individual living in a household using a specific place for waste disposal.

$\ddagger$ Protective effectiveness is adjusted for individual living in a household using a specific place for waste disposal. 
consecutive days, heart rate $\leq 80$ beats per minute, and palpable spleen) for whom ViPS vaccine was $80 \%$ efficacious; however, specificity or sensitivity of these criteria was not reported. ${ }^{49}$ When we examined these criteria from the Nepal trial for culture-negative typhoid in our data, we found that there were four episodes in HepA vaccinees versus zero episodes in ViPS recipients. To our knowledge, this is the first study to offer a validated clinical predictor rule to diagnose typhoid using clinical data.

Our findings should be interpreted in light of several important limitations. First, reporting of clinical signs and symptoms may have suffered from errors due to limitations in the accuracies of medical histories provided by patients and the examinations performed by clinicians under hectic outpatient clinic conditions in crowded study facilities in urban slums. However, our findings do provide a realistic picture of diagnostic rules that can be used in such settings, which are common in typhoid-endemic populations in the developing world. Second, we did not consider point-of-care diagnostic laboratory results, as these were not uniformly available at the time of presentation in the clinics. Again, the absence of availability of such data for clinical decision-making at the time of patient presentation may be the rule, not the exception, in typhoid-endemic settings. Third, we did not include any postpresentation clinical information-which would not be applicable to the needs of clinicians making decisions on initial presentation of febrile patients, but might be of interest for other purposes, such as classifying BC-negative typhoid end points for a typhoid vaccine efficacy trial. Fourth, our analysis contained a mix of vaccinated and unvaccinated febrile patients. In future analyses, we will consider the applicability of our clinical prediction rule to these two types of patients separately. Fifth, it is possible that our findings are idiosyncratic to the population and epidemiological setting for the study. Further assessments in disparate populations are required to determine whether clinical predictors might be generalizable from one setting to another. Finally, the level of protective effectiveness observed in the Vi probe analysis in the training and validation sets, although statistically significant and consistent between the two sets, was lower than the $61 \%$ protection by $\mathrm{Vi}$ against BC-proven typhoid in the Kolkata trial. This could mean that our rules for clinical typhoid were insufficiently specific and that Vi protection is indeed lower against BC-negative typhoid fever, or both.

However, several considerations strengthen our findings. First, the study was a large, prospective, population-based cohort, comprehensively followed and systematically evaluated for febrile episodes and for typhoid using well-validated microbiological methods in experienced clinical laboratories. Second, the accumulated data were sufficient in size to make use of independent cohorts to both derive and test the clinical prediction rule. Moreover, validation of the derived rule was strengthened by demanding high diagnostic specificity and using the results of a vaccine probe analysis. Finally, vaccine protection against all persistent fever episodes was not detected, demonstrating the discriminatory value of our predictive rule.

In the absence of a sensitive typhoid point-of-care diagnostic, a reliable and highly specific clinical rule for predicting typhoid, irrespective of culture positivity, will be useful to guide healthcare workers practicing in endemic, low-resource settings. ${ }^{50}$ With a new series of phase III and IV trials being undertaken in Bangladesh, Nepal, and Malawi to evaluate new generation,
Vi-protein conjugate vaccines, ${ }^{16,51-53}$ our vaccine probe approach should be considered in future analyses to confirm and extend our findings.

Received December 26, 2019. Accepted for publication May 4, 2020.

Published online June 22, 2020.

Note: Supplemental table appears at www.ajtmh.org.

Financial support: The research was supported by the Diseases of the Most Impoverished Program of the Bill \& Melinda Gates Foundation. This publication was made possible through a grant from the Bill \& Melinda Gates Foundation (OPP1171432).

Authors' addresses: Md. Taufiqul Islam, Faisal Ahmmed, Ashraful Islam Khan, Khalequ Zaman, and Firdausi Qadri, International Centre for Diarrheal Disease Research, Bangladesh, Dhaka, Bangladesh, E-mails: taufiqulislam@icddrb.org, faisal.ahmmed@icddrb.org, ashrafk@icddrb.org, kzaman@icddrb.org, and fqadri@icddrb.org. Justin Im, Deok Ryun Kim, and Jerome H. Kim, International Vaccine Institute, Seoul, Republic of Korea, E-mails: justin.im@ivi.int, drkim@ivi.int, and jerome.kim@ivi.int. Mohammad Ali, Johns Hopkins University, Baltimore, MD, E-mail: mali25@jhu.edu. Florian Marks, International Vaccine Institute, Seoul, Republic of Korea, and Department of Medicine, University of Cambridge, Cambridge, United Kingdom, E-mail: fmarks@ivi.int. John D. Clemens, International Centre for Diarrheal Disease Research, Bangladesh, Dhaka, Bangladesh, UCLA Fielding School of Public Health, Los Angeles, CA, and Korea University College of Medicine, Seoul, South Korea, E-mail: jclemens@icddrb.org.

This is an open-access article distributed under the terms of the Creative Commons Attribution (CC-BY) License, which permits unrestricted use, distribution, and reproduction in any medium, provided the original author and source are credited.

\section{REFERENCES}

1. GBD 2017 Typhoid and Paratyphoid Collaborators, 2019. The global burden of typhoid and paratyphoid fevers: a systematic analysis for the Global Burden of Disease Study 2017. Lancet Infect Dis 19: 369-381.

2. Buckle GC, Walker CL, Black RE, 2012. Typhoid fever and paratyphoid fever: systematic review to estimate global morbidity and mortality for 2010. J Global Health 2: 010401.

3. Crump JA, Sjolund-Karlsson M, Gordon MA, Parry CM, 2015. Epidemiology, clinical presentation, laboratory diagnosis, antimicrobial resistance, and antimicrobial management of invasive Salmonella infections. Clin Microbiol Rev 28: 901-937.

4. Crump JA, Luby SP, Mintz ED, 2004. The global burden of typhoid fever. Bull World Health Organ 82: 346-353.

5. Antillon M, Saad NJ, Baker S, Pollard AJ, Pitzer VE, 2018. The relationship between blood sample volume and diagnostic sensitivity of blood culture for typhoid and paratyphoid fever: a systematic review and meta-analysis. J Infect Dis 218 (Suppl_4): S255-S267.

6. Storey HL, Huang Y, Crudder C, Golden A, de los Santos T, Hawkins K, 2015. A meta-analysis of typhoid diagnostic accuracy studies: a recommendation to adopt a standardized composite reference. PLoS One 10: e0142364.

7. Mogasale V, Ramani E, Mogasale VV, Park J, 2016. What proportion of Salmonella Typhi cases are detected by blood culture? A systematic literature review. Ann Clin Microbiol Antimicrob 15: 32

8. Vollaard AM, Ali S, Widjaja S, Asten HA, Visser LG, Surjadi C, van Dissel JT, 2005. Identification of typhoid fever and paratyphoid fever cases at presentation in outpatient clinics in Jakarta, Indonesia. Trans R Soc Trop Med Hyg 99: 440-450.

9. Arora P, Thorlund K, Brenner DR, Andrews JR, 2019. Comparative accuracy of typhoid diagnostic tools: a Bayesian latent-class network analysis. PLoS Negl Trop Dis 13: e0007303.

10. Parry CM, 2003. Antimicrobial drug resistance in Salmonella enterica. Curr Opin Infect Dis. 16: 467-472.

11. Holt KE et al., 2011. Emergence of a globally dominant IncHI1 plasmid type associated with multiple drug resistant typhoid. PLoS Negl Trop Dis 5: e1245. 
12. Akhtar S, Sarker MR, Jabeen K, Sattar A, Qamar A, Fasih N, 2015. Antimicrobial resistance in Salmonella enterica serovar typhi and paratyphi in south Asia-current status, issues and prospects. Crit Rev Microbiol 41: 536-545.

13. Darton TC et al., 2016. Using a human challenge model of infection to measure vaccine efficacy: a randomised, controlled trial comparing the typhoid vaccines $\mathrm{M} 01 \mathrm{ZH} 09$ with placebo and Ty21a. PLoS Negl Trop Dis 10: e0004926.

14. Jin C et al., 2017. Efficacy and immunogenicity of a Vi-tetanus toxoid conjugate vaccine in the prevention of typhoid fever using a controlled human infection model of Salmonella Typhi: a randomised controlled, phase $2 \mathrm{~b}$ trial. Lancet 390: 2472-2480.

15. Mohan VK, Varanasi V, Singh A, Pasetti MF, Levine MM, Venkatesan R, Ella KM, 2015. Safety and immunogenicity of a Vi polysaccharide-tetanus toxoid conjugate vaccine (Typbar-TCV) in healthy infants, children, and adults in typhoid endemic areas: a multicenter, 2-cohort, open-label, double-blind, randomized controlled phase 3 study. Clin Infect Dis 61: 393-402.

16. Meiring JE, Gibani M; TyVAC Consortium Meeting Group, 2017. The Typhoid Vaccine Acceleration Consortium (TyVAC): vaccine effectiveness study designs: accelerating the introduction of typhoid conjugate vaccines and reducing the global burden of enteric fever. Report from a meeting held on 26-27 October 2016, Oxford, UK. Vaccine 35: 5081-5088.

17. Carey ME, Diaz ZI, Broadstock M, Bailey R, Bentsi-Enchill AD, Larson HJ, 2019. Toward control? The prospects and challenges of typhoid conjugate vaccine introduction. Clin Infect Dis 69 (Suppl_5): S408-S411.

18. Gessner BD et al., 2005. Incidences of vaccine-preventable Haemophilus influenzae type $\mathrm{b}$ pneumonia and meningitis in Indonesian children: hamlet-randomised vaccine-probe trial. Lancet 365: 43-52.

19. Gessner BD, Sedyaningsih ER, Griffiths UK, Sutanto A, Linehan M, Mercer D, Mulholland EK, Walker DG, Steinhoff M, Nadjib M, 2008. Vaccine-preventable Haemophilus influenza type B disease burden and cost-effectiveness of infant vaccination in Indonesia. Pediatr Infect Dis J 27: 438-443.

20. Gessner BD, Brooks WA, Neuzil KM, Vernet G, Bright RA, Tam JS, Bresee J, Monto AS, 2013.Vaccines as a tool to estimate the burden of severe influenza in children of low-resourced areas (November 30-December 1, 2012, Les Pensieres, Veyrier-du-Lac, France). Vaccine 31: 3222-3228.

21. Gessner BD, Halloran ME, Khan I, 2015. The case for a typhoid vaccine probe study and overview of design elements. Vaccine 33 (Supp/ 3): C30-C35.

22. Feikin DR, Scott JA, Gessner BD, 2014. Use of vaccines as probes to define disease burden. Lancet 383: 1762-1770.

23. Sur $\mathrm{D}$ et al., 2009. A cluster-randomized effectiveness trial of $\mathrm{Vi}$ typhoid vaccine in India. N Engl J Med 361: 335-344.

24. Keddy $\mathrm{KH}$, Sooka A, Letsoalo ME, Hoyland G, Chaignat CL, Morrissey AB, Crump JA, 2011. Sensitivity and specificity of typhoid fever rapid antibody tests for laboratory diagnosis at two sub-Saharan African sites. Bull World Health Organ 89: 640-647.

25. Akoh JA, 1991. Relative sensitivity of blood and bone marrow cultures in typhoid fever. Trop Doct 21: 174-176.

26. Parry CM, Wijedoru L, Arjyal A, Baker S, 2011. The utility of diagnostic tests for enteric fever in endemic locations. Expert Rev Anti infect Ther 9: 711-725.

27. Wain J, Hosoglu S, 2008. The laboratory diagnosis of enteric fever. J Infect Dev Ctries 2: 421-425.

28. Baker S, Sarwar Y, Aziz H, Haque A, Ali A, Dougan G, Wain J, Haque A, 2005. Detection of Vi-negative Salmonella enterica serovar typhi in the peripheral blood of patients with typhoid fever in the Faisalabad region of Pakistan. J Clin Microbiol 43: 4418-4425.

29. Strobl C, Malley J, Tutz G, 2009. An introduction to recursive partitioning: rationale, application, and characteristics of classification and regression trees, bagging, and random forests. Psychol Methods 14: 323-348.

30. Bopp CABF, Wells JG, Strockbine NA, 1999. Escherichia, shigella and salmonella. Murray PR, ed. Manual of Clinical Biology, 7th edition. Washington, DC: ASM Press.
31. Oonsivilai M, Mo Y, Luangasanatip N, Lubell $\mathrm{Y}$, Miliya $\mathrm{T}$, Tan $\mathrm{P}$, Loeuk L, Turner P, Cooper BS, 2018. Using machine learning to guide targeted and locally-tailored empiric antibiotic prescribing in a children's hospital in Cambodia. Wellcome Open Res 3: 131.

32. Schaffer C, 1993. Selecting a classification method by crossvalidation. Mach Learn 13: 135-143.

33. Hand DJ, 2009. Measuring classifier performance: a coherent alternative to the area under the ROC curve. Mach Learn 77: 103-123.

34. Faraggi $D$, Reiser $B, 2002$. Estimation of the area under the ROC curve. Stat Med 21: 3093-3106.

35. Amorim LD, Cai J, 2015. Modelling recurrent events: a tutorial for analysis in epidemiology. Int J Epidemiol 44: 324-333.

36. Robin X, Turck N, Hainard A, Tiberti N, Lisacek F, Sanchez JC, Müller M, 2011. pROC: an open-source package for R and S+ to analyze and compare ROC curves. BMC Bioinf 12: 77.

37. Terry M, Therneau PMG, 2000. Modeling Survival Data: Extending the Cox Model. New York, NY: Springer.

38. Wickham H, François R, Henry L, Müller K, 2020. dplyr: A Grammar of Data Manipulation. R Package Version 0.7.6. https:// CRAN.R-project.org/package $=$ dplyr.

39. Gilli M, Maringer D, Schumann E, 2011. Numerical Methods and Optimization in Finance. Cambridge, MA: Academic Press.

40. Sinha A, Sazawal S, Kumar R, Sood S, Reddaiah VP, Singh B, Rao M, Naficy A, Clemens JD, Bhan MK, 1999. Typhoid fever in children aged less than 5 years. Lancet 354: 734-737.

41. Siddiqui FJ, Rabbani F, Hasan R, Nizami SQ, Bhutta ZA, 2006. Typhoid fever in children: some epidemiological considerations from Karachi, Pakistan. Int J Infect Dis 10: 215-222.

42. Walia M, Gaind R, Mehta R, Paul P, Aggarwal P, Kalaivani M, 2005. Current perspectives of enteric fever: a hospital-based study from India. Ann Trop Paediatr 25: 161-174.

43. Papaevangelou V, Syriopoulou V, Charissiadou A, Pangalis A, Mostrou G, Theodoridou M, 2004. Salmonella bacteraemia in a tertiary children's hospital. Scand J Infect Dis 36: $547-551$

44. Ross IN, Abraham T, 1987. Predicting enteric fever without bacteriological culture results. Trans $\mathrm{R}$ Soc Trop Med Hyg 81: 374-377.

45. Matono $\mathrm{T}$, Kutsuna $\mathrm{S}$, Kato $\mathrm{Y}$, Katanami $\mathrm{Y}$, Yamamoto $\mathrm{K}$, Takeshita N, Hayakawa K, Kanagawa S, Kaku M, Ohmagari N, 2017. Role of classic signs as diagnostic predictors for enteric fever among returned travellers: relative bradycardia and eosinopenia. PLoS One 12: e0179814.

46. Haq SA, Alam MN, Hossain SM, Ahmed T, Tahir M, 1997. Value of clinical features in the diagnosis of enteric fever. Bangladesh Med Res Counc Bull 23: 42-46.

47. Hosoglu S, Geyik MF, Akalin S, Ayaz C, Kokoglu OF, Loeb M, 2006. A simple validated prediction rule to diagnose typhoid fever in Turkey. Trans R Soc Trop Med Hyg 100: 1068-1074.

48. Khan M, Coovadia YM, Connoly C, Sturm AW, 1998. The early diagnosis of typhoid fever prior to the widal test and bacteriological culture results. Acta Trop 69: 165-173.

49. Acharya IL et al., 1987. Prevention of typhoid fever in Nepal with the $\mathrm{Vi}$ capsular polysaccharide of Salmonella typhi. A preliminary report. N Engl J Med 317: 1101-1104.

50. Parry CM, Hien TT, Dougan G, White NJ, Farrar JJ, 2002. Typhoid fever. N Engl J Med 347: 1770-1782.

51. Jamka LP, Simiyu KW, Bentsi-Enchill AD, Mwisongo AJ, Matzger H, Marfin AA, Pollard AJ, Neuzil KM, 2019. Accelerating typhoid conjugate vaccine introduction: what can be learned from prior new vaccine introduction initiatives? Clin Infect Dis 68 (Suppl_2): S171-S176.

52. Meiring JE et al., 2019. Typhoid vaccine acceleration consortium Malawi: a phase III, randomized, double-blind, controlled trial of the clinical efficacy of typhoid conjugate vaccine among children in Blantyre, Malawi. Clin Infect Dis 68 (Supple_2): S50-S58.

53. Theiss-Nyland $\mathrm{K}$ et al., 2019. Assessing the impact of a Vipolysaccharide conjugate vaccine in preventing typhoid infection among Bangladeshi children: a protocol for a phase Illb trial. Clin Infect Dis 68 (Supple_2): S74-S82. 